\title{
Measuring the perception of light inconsistencies
}

\author{
Jorge Lopez-Moreno $^{1} \quad$ Veronica Sundstedt $^{2,3} \quad$ Francisco Sangorrin $^{1} \quad$ Diego Gutierrez $^{1}$ \\ ${ }^{1}$ Universidad de Zaragoza $\quad{ }^{2}$ Blekinge Institute of Technology $\quad{ }^{3}$ Trinity College Dublin
}

\begin{abstract}
In this paper we explore the ability of the human visual system to detect inconsistencies in the illumination of objects in images. We specifically focus on objects being lit from different angles as the rest of the image. We present the results of three different tests, two with synthetic objects and a third one with digitally manipulated real images. Our results agree with previous publications exploring the topic, but we extend them by providing quantifiable data which in turn suggest approximate perceptual thresholds. Given that light detection in single images is an ill-posed problem, these thresholds can provide valid error limits to related algorithms in different contexts, such as compositing or augmented reality.
\end{abstract}

CR Categories: I.3.7 [Computing Methodologies]: Computer Graphics-3D Graphics; I.4.10 [Computing Methodologies]: Image Processing and Computer Vision-Image Representation

\section{Introduction}

The process of perception in the human visual system (HVS) is a complex phenomenon which starts with the formation of an image in the retina. This image is subsequently analyzed and processed by the HVS in order to extract significative data while disregarding unnecessary information.

Areas such as computer graphics deal with the creation of images by simulating the complex interactions of light and matter in its path towards the retina. However, if we disregard the remaining part of the perception process it is likely that most of these computations could have been avoided. For instance, JPEG format achieves great image compression ratios by removing frequencies which are not easily perceived by the HVS.

Multiple technologies like augmented reality [Wang and Samaras 2002; Zhang and Yang 2001], image editing [Yu et al. 2006] or image forensics [Johnson and Farid 2005; Johnson and Farid 2007] strongly rely in the process of detecting the lighting environment and inserting new objects relit in the same fashion as their neighbors. For this, the ability to estimate the light direction in the original scene becomes a crucial step. This can be done in controlled environments, but when there is limited information (like in a single image), this task becomes difficult or simply impossible. The influence of shape, material or lighting becomes integrated into a single pixel value and disambiguating this information is not possible without any prior information. This may be further complicated due to uncontrolled factors in the input images such as lens distortion or glare. In these uncontrolled environments, light detection algorithms are expected to yield large errors in their estimations. However, these errors might go completely unnoticed by users in an image while they are easily spotted in another.
In this work we are interested in determining an error threshold below which variations in the direction vector of the lights will not be noticed by a human observer. To this end we performed a set of psychophysical experiments where we analyze several factors involved in the general light detection process, while measuring their degree of influence for its future use in computer applications.

There are several aspects involved in the process of light detection. For example the object material, texture frequency, the presence of visual cues such as shadows, light positions and the level of user training are all relevant. The most frequent scenarios to acquire a useful measure studied in present tests have focused on different aspects. Work by Ostrovsky et al. [2005] studied the influence of the light positions. They anticipated that a greater presence of shadows (produced when the light source is behind the object) increases the accuracy of the HVS.

Our overall goal is to obtain a valid range of values in which the HVS is not able to distinguish lighting errors in very general scenarios. Scenarios we would like to consider are scenes with multiple light sources and material properties and a complete range of light positions. It is important to notice that all our tests preclude the presence of strong visual shadow cues in horizontal surfaces by the objects of the scene. These scenes were excluded based on two main reasons: (1) the subject has been studied in great depth in previous work and its influence has been clearly stated and more importantly (2) it is a visual cue that might not be present in many scenarios in opposition to shading, materials, or self shadowing which are ever-present features.

\section{Related Work}

Todd and Mingolla [1983] showed the low accuracy of the HVS in determining the light direction by observing a lightprobe. They stated that the presence of highlights did not help in the estimation of the illuminant's direction. However, their measures were limited to cylinders (a simple geometry which varies in only one axis) and the users were asked for the direction of light (the inverse of the present case). In the same line, the same authors disproved the general belief that the HVS assumes objects as diffuse by default [Mingolla and Todd 1986].

Additionally Koenderik et al. [2004] showed how human perception is much better at azimuth estimates than at zenith estimates. They also proved that when shadows are present, the shadow boundaries (a first order discontinuity in shading) increased the accuracy of HVS in detecting the light field direction.

Previous research has shown that the visual system assumes that light is coming from above and slightly to the left of a shaded object [Sun and Perona 1998; Mamassian and Goutcher 2001]. A recent work by O'Shea et al. [2008] confirmed this light-from-above prior and provided the quantifiable evidence that for unknown geometries the angle between the viewing direction and the light direction is assumed to be 20-30 degrees above the viewpoint. Ostrovsky et al. [2005] show that humans can easily spot an anomalously lit object in an array of identical objets with the same orientation and lit exactly the same, but performance drops when altering orientations of the equally-lit objects. In a similar manner, in this work we aim to extend previous results [Ostrovsky et al. 2005] by providing a 


\begin{tabular}{|l|c|c|c|c|c|c|c|c|}
\hline & a & b & c & d & e & f & g & h \\
\hline Diffuse & Yes & Yes & Yes & No & No & Yes & No & No \\
Textured & No & P(h) & No & CHK & CHK & No & P(l) & No \\
\hline
\end{tabular}

Table 1: Description of materials per object $(a-h)$ shown in the images of the test. The top row indicates if the material is only diffuse, otherwise it has a highly specular (Phong) reflectance. $\boldsymbol{P}(\boldsymbol{h})$ and $\boldsymbol{P}(\boldsymbol{l})$ describe a texture obtained through Perlin's Noise at different spatial scales (high and low frequency respectively) and $\boldsymbol{C H K}$ corresponds to a black and white checkerboard texture.

wider set of scenarios, adding eye tracking data and quantifying the results. We first present an extension of the experiments made by Lopez-Moreno et al. [2009]. Secondly, we analyze the influence of light position adding new insights by analyzing eye tracking data. Finally, we present two new experiments which analyze the influence of texture frequency and extrapolate our findings to real-world images, respectively.

\section{Experiment One: Overall Inaccuracy}

In the first experiment our goal is to check how capable the human visual system is of spotting illumination errors in three different lighting situations. Images with several objects are shown (see Figure 1), all of them lit from the same angle, except for one, which is lit with a varying degree of divergence with respect to the rest. We limit the study to the more restrictive case of the azimuth angle, according to previous findings [Koenderink et al. 2004].

Four of the objects have no texture, two have high-frequency and two have low-frequency textures. Four of the objects are shiny, while four are diffuse. Table 1 summarizes their characteristics. The motivation of the scene and the diversity of materials is chosen to represent a wide enough range. In particular, the shape of the objects has been chosen to be abstract in order to avoid semantical significance and globally convex (according to global convexity default assumption of the HVS [Langer and Bülthoff 2001]). They have a relatively complex surface, but with limited variance (to avoid the influence of geometry [Vangorp et al. 2007]) and are arranged to avoid direct side-by-side comparisons of exactly equal geometries.

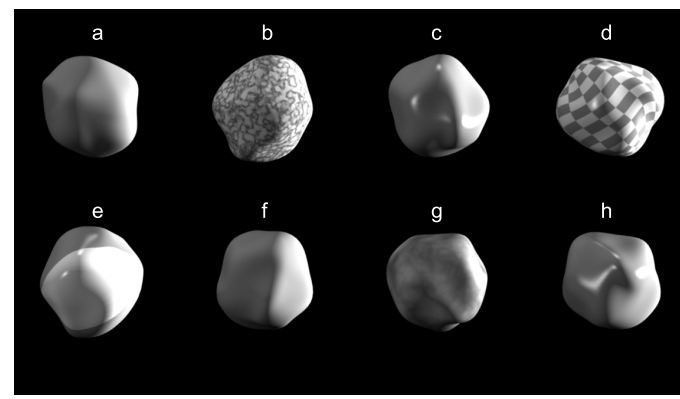

Figure 1: Example image for our first experiment: eight abstract objects with a main light coming from the right.

We consider the $\mathrm{Y}$ axis as the vertical axis of the screen plane $\mathrm{XY}$ and $\mathrm{Z}$ as the positive XY-plane direction. In each of the 60 images, all the objects are illuminated with an ambient light made up by two directional sources. One is located at 45 degrees between the axis $+\mathrm{Z}$ and the axis $-\mathrm{X}$ and the other situated on top of the axis $\mathrm{Y}$. Their intensities are four times weaker in terms of luminance than the main light. This main light is also a directional light and is the same for seven of the eight objects, while the eighth is lit from a different direction. Thus, we will refer to the these as the two main lights in the image: the "correct" one, illuminating seven objects and the "wrong" one, illuminating the eighth.

The two main lights vary their angle $\phi$ along the $\mathrm{XZ}$ plane between different images (top row in Figure 2). The absolute difference in $\phi$ between the two directional lights increases from $0^{\circ}$ to a maximum difference of $90^{\circ}$ in $10^{\circ}$-increments $\left(5^{\circ}\right.$ in each angular direction). We thus obtain ten test images. To further analyze the influence of light direction, we repeat this procedure with three different situations: First with both sources illuminating the frontal hemisphere of the object, secondly with both sources illuminating from behind the object and finally with one light coming from the back and the other from the front (Figure 2).

Half the times a shiny object is incorrectly lit and the other half a diffuse object is incorrectly lit. There are thus 60 images in total (10 increasing degrees of divergence, times three light configurations, times two types of inconsistently lit objects), each showing eight asymmetrical objects with different textures and degrees of shininess. Each image has a resolution of 1024 pixels wide by 600 pixels high. The order in that images were displayed was randomized, as well as the object that was inconsistently lit in each image. The test was performed through a web application, where users were asked, after an introductory explanation, to simply select the inconsistently lit object in each image. Although the time it takes each participant to complete the test is measured, there is no limitation in that regard. 55 participants took the test (ages 16-58; 33 male, 22 female), 18 of which had an artistic background.

\subsection{Results}

We analyze the number of correct answers (which we term hits) depending on the difference between the two lights for the two material cases: diffuse and shiny, according to the different configuration of lights (Figure 3 ). We can observe that up to 20 degrees of divergence the probability of detection is around chance (12.5\%). In the case that both lights are in the front this probability keeps on being below chance up to 30 degrees. On the contrary when the lights are at the back the probability of detection is higher at 20 degrees of divergence. This seems to agree with previous studies [Koenderink et al. 2004], suggesting that shaded areas and self-shadows increase our accuracy inferring light directions from images.

Furthermore, we can observe that for any position of the light source, the performance of HVS is slightly lower when highlights are present. Although further analysis should be carried out to find out why highlights have an apparently negative effect, this seems to agree with Todd and Mingolla's [1983] previous work, which diverges from some computer vision approaches which do use highlights as visual cues [Lagger and Fua 2006].

We found no statistical difference across genders for this particular task, as opposed to other tasks like mental rotation, which has shown different reasoning strategies per gender [Hugdahl et al. 2006]. Our results also showed that participants with an artistic background had significantly better results at judging light directions, achieving about $15 \%$ more correct answers on average.

Regarding the time spent per image, the average was 15.13 seconds. For the diffuse material, as expected, times were shorter as the error increased, meaning it was easier to spot (see Figure 4). However, the trend is less obvious in the presence of highlights: again, highlights seem to play a negative role for this particular task that is worth studying further.

Object saliency : Amongst the 60 images there are six control images (0-degree divergence) in which all objects are illuminated 

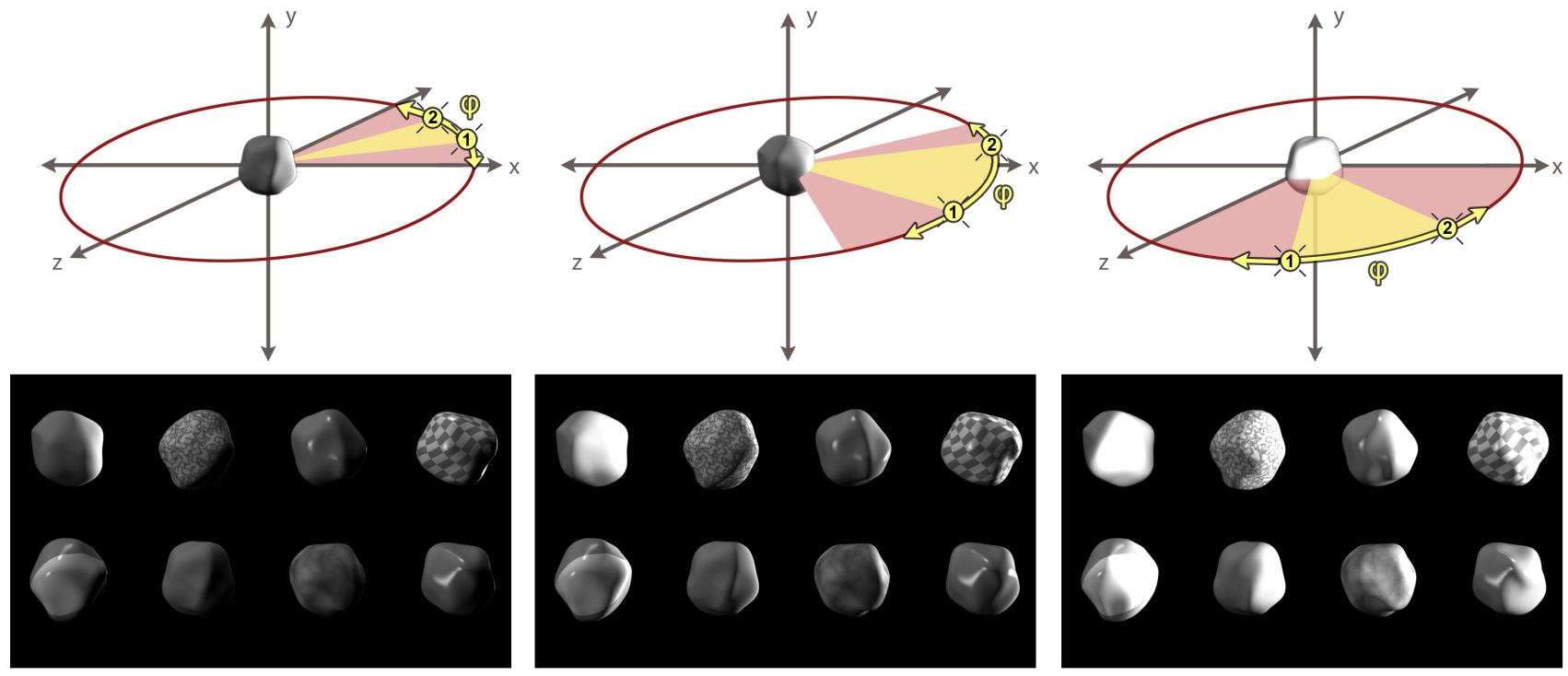

Figure 2: Top Row: 3D representation of the scenes rendered in our images. Light 2 is the global light of the scene and light number 1 is the "wrong" light affecting a single object. The angular divergence of the direction of the two light sources is shown in yellow for the case of $60^{\circ}$-divergence, while the maximum $90^{\circ}$-divergence is displayed in red for each case. Bottom Row: the correspondingly lit objects.
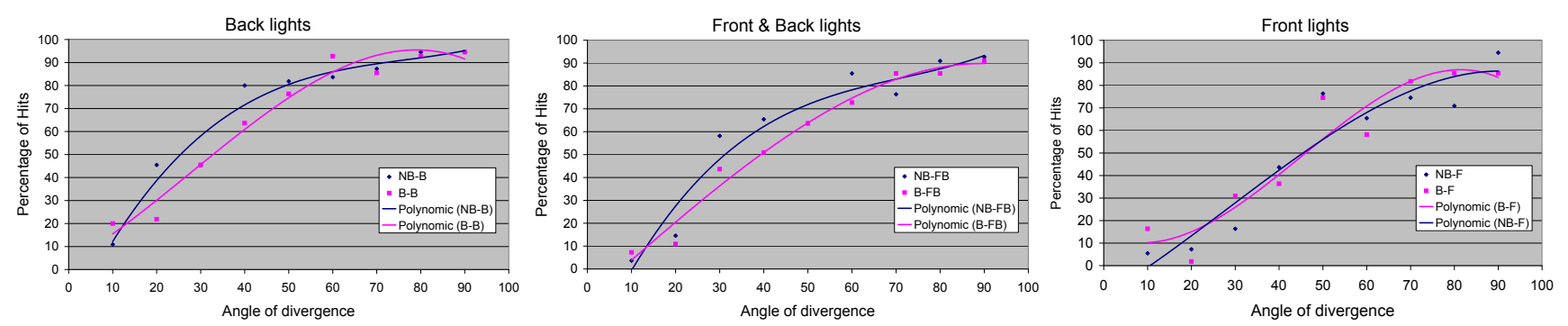

Figure 3: Hit probability by quadrant for both shiny (B, pink) and diffuse (NB, blue) materials. Left: with frontal position. Middle: with back position. Right: with front-back position.

correctly; this can help us detect potential salient objects. Figure 5 shows a bar chart with the different options that users have selected for these images. Each of the three bars corresponds to the three positions of the lights (both lights behind the object predominating the shadows versus the lights, one front and one back and two lights in the front, predominating the lights versus the shadows). It is interesting to notice that there is a clear outlier, object $E$, probably due to its particular geometry and white albedo patch. In the chart of Figure 5 we can observe how its salience compared with the remaining objects is reduced in direct relation with the increase of divergence. In other words, for low or no divergence in light direction, object $E$ was selected due to salient features outside the purpose of this test. But as the degrees of divergence increase, its saliency becomes less apparent due to the presence of a clearly incorrectly-lit object.

Additionally, five users were shown the same series of images as in our previous test, but in this case they were not given any specific task and were asked just to observe the images during a limited time, which was set to 15 seconds based on the average time per question of the previous test. We divided each image in eight regions of interest (ROI) corresponding to the eight synthetic objects and tracked their average eye fixation time in order to analyze the evolution of salience per object.

From the resulting heat maps (see Figure 6), we can analyze the gradient of the salience for a incorrectly lit object. This can be done due to the design of this test: the inconsistently lit objects alternate between being incorrectly lit and being illuminated as the rest. For instance, at 10 degrees of divergence $\mathrm{F}$ is inconsistently lit and $\mathrm{A}$ is correct while for 20 degrees $\mathrm{A}$ is correct and $\mathrm{F}$ is wrong, etc. Figure 7 shows the results, where an overall alternancy in saliency can be observed, as expected. However, more experiments need to be carried out to disambiguate other factors such as highlights, texture and geometry.

\section{Experiment Two: Texture Influence}

In this experiment we aim to analyze the influence in the perception process of the spatial frequency of the texture. The psychophysical test consists of a new series of images, which has been shown to 32 users (ages 22-57; 23 male and 9 female). The test was displayed using the same methodology as in Experiment One.

We analyze four different checkerboard textures of increasing spatial frequency (which we term low, medium, medium-high and high. 

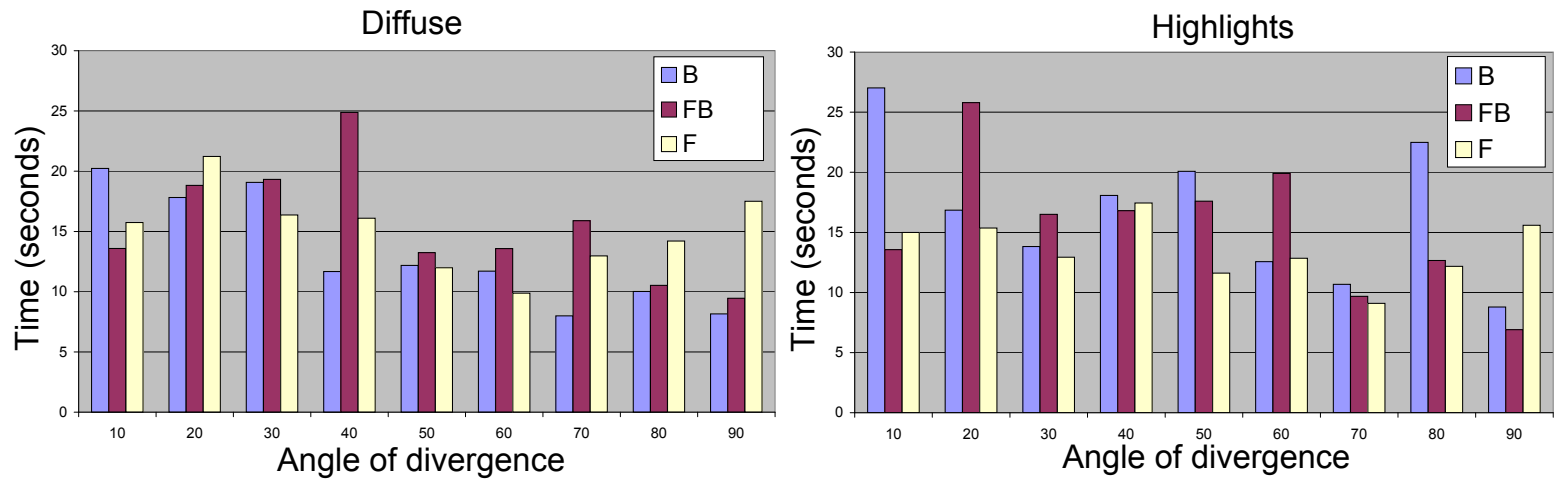

Figure 4: Time used to make decisions in our test, shown by increasing divergence and grouped by quadrant: Front (F), back (B) and front-back $(F B)$. Please note that the questions were randomized and this is not a trend produced by fatigue or training.
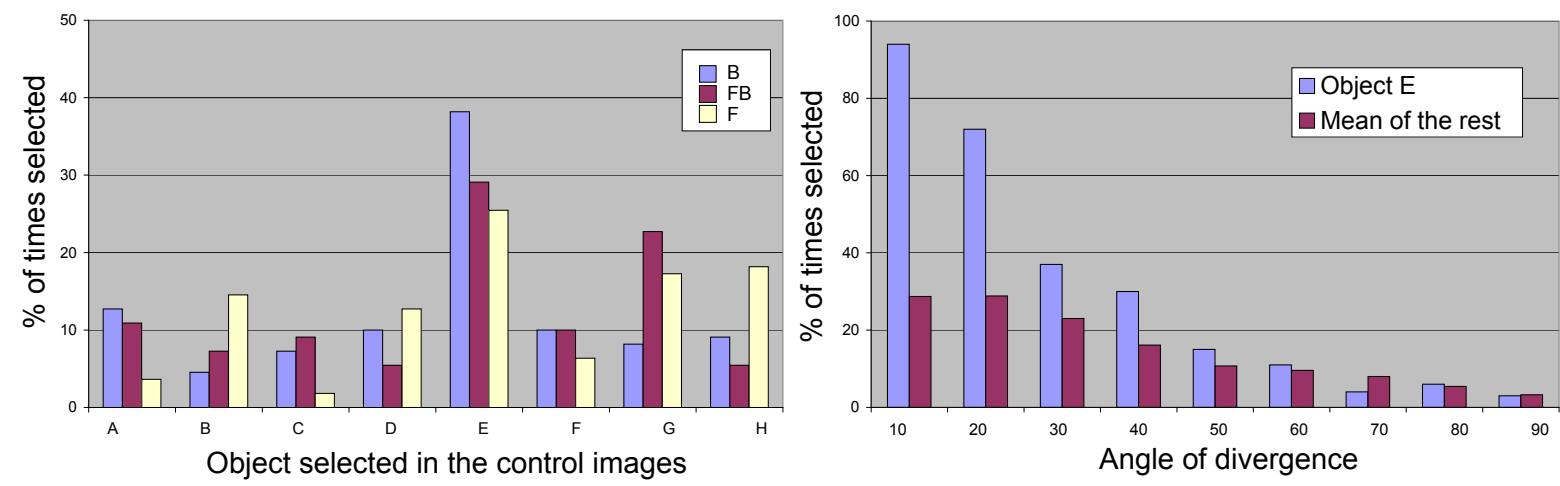

Figure 5: Left:Chosen object in the control images, grouped by quadrant: Front $(F)$, back $(B)$ and front-back $(F B)$. The users have a preference for object E. Right: The relative salience of the object E, computed as the number of times when it is chosen while missing the right choice. This is plotted in relation with the salience of the remaining objects.
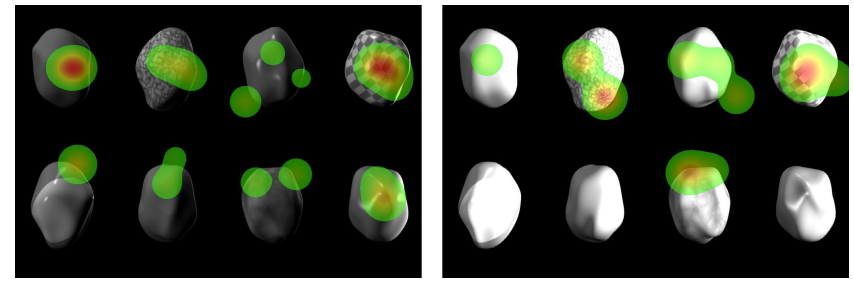

Figure 6: Example of heat maps representing average fixation time at two images for one user.

Each one has a tile size two times smaller than the previous one. We do not aim to explore the luminance frequency, instead we fix the luminance ratio between the two albedos so that shading cue is always perceivable. With this configuration [Adelson and Pentland 1996], the luminance of a clear tile in shadows is similar to the luminance of a dark tile in a lit area (See Figure 8). The shininess of the material is set to a $50 \%$ of the value used for shiny objects in the previous test. This is done in order to analyze the results. The shape of the curve should fit between the curves for diffuse and shiny objects of the previous test.

Each user observes a series of 40 images (4 textures x 10 divergence values) with lights being modified in the same fashion as in our previous test. In order to reduce dimensionality, we limit the movement of the lights to the front-back quadrant. For each image,

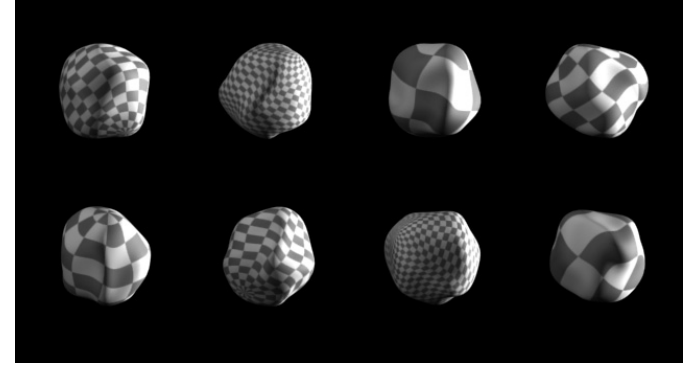

Figure 8: An example of an image used in our test. Four different texture patterns are assigned to eight random objects.

a random object is selected to be inconsistently lit (with a certain texture) and for the remaining objects both the textures and the geometries are randomly set.

\subsection{Results}

In Figure 9 we can observe a similar curve as in the first experiment, with some differences for the four textures. From the data collected, it seems that higher frequencies do mask lighting inaccuracies up to the detection threshold of 20-30 degrees, making the detection task more difficult. For divergence angles above 40 degrees we found 

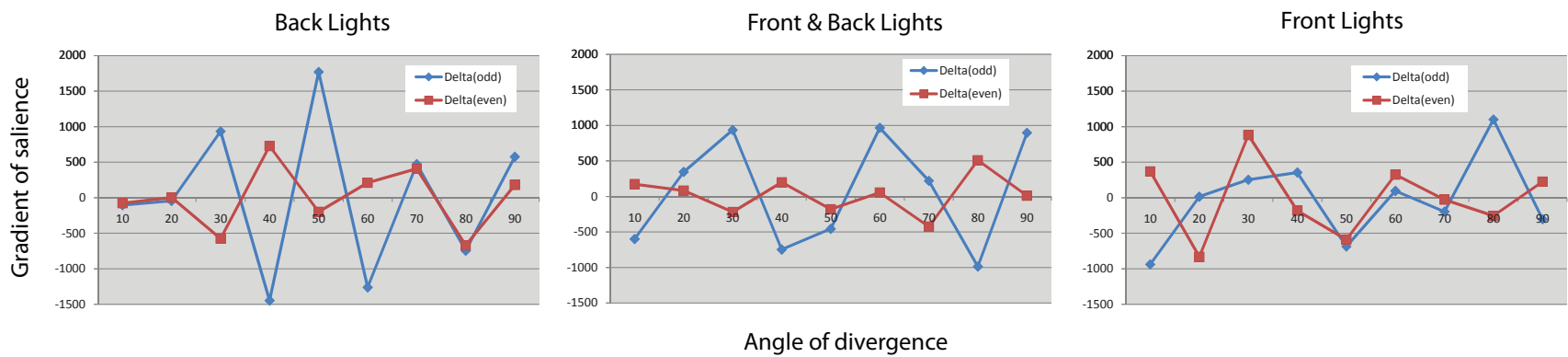

Figure 7: Gradient of the ratio between time spent watching the reiluminated object and the average time spent watching the rest of objects. At each graph, object $A$ is represented in red (inconsistently lit at 20, 40, 60 and 80) and object $F$ is represented in blue (inconsistently lit at $10,30,40,50,70$ and 90 ).

no significant difference $(p>0.05)$ in the results. This shows that, at least for the pattern shown and the frequencies used, no amount of high frequency texture information can mask large inaccuracies in low frequency lighting information. This seems to coincide with the results of Khang et al. [2006] which suggest that the visual system may not take intensity variations due to the surface material or the light field into account when estimating the direction of illumination. We find an interesting line of future work in analyzing the transition area from masking to non-masking effects of the texture and the interplay between high and low frequency information in an image.

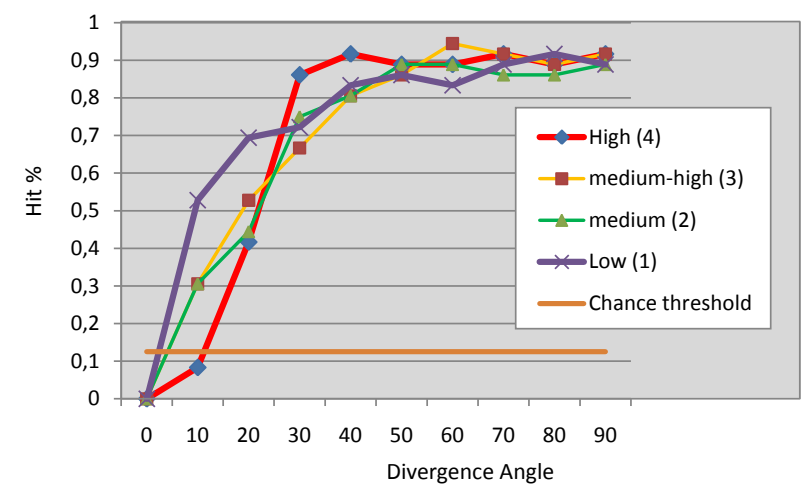

Figure 9: Statistics of the responses provided by users in the test, shown by texture frequency.

\section{Experiment Three: Real World Images}

In order to explore how well our findings carry over into real images, we have run two additional experiments with modified photographs as stimulus. The display methodology was based on the same web test as in previous experiments.

Experiment 3.1 : The first test consists of a simple scene containing a set of eight real objects (see Figure 10). The scene was photographed three times: the original scene, plus two more with the angle of the main light source varying 20 and 30 degrees respectively. Two objects from the original image were replaced by their counterparts from the two images with varying light sources. They were composited on top of the original image: the ceramic purple doll and the Venus figurine, both having diffuse and specular components and near-constant albedos. We thus create two "real world" equivalents of objects inconsistently lit, as in our first two experiments: one image with two objects incorrectly lit at 20 degrees and a second one at 30 degrees.
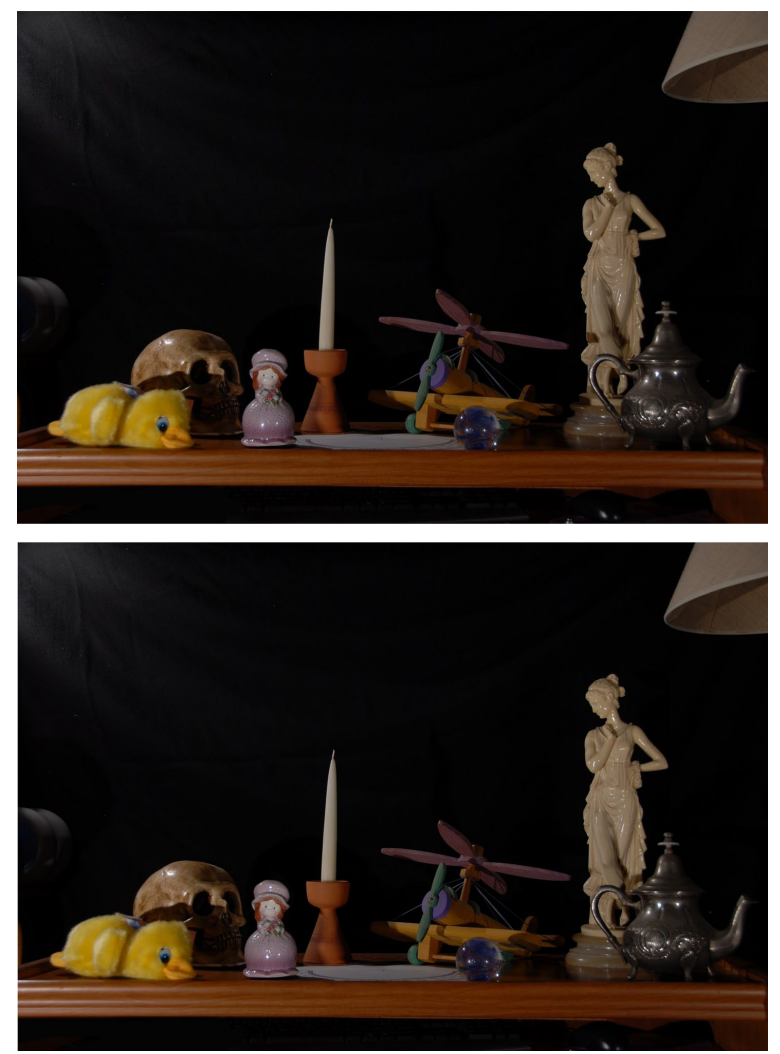

Figure 10: Image used in our test, in which the doll and the statue of Venus have been reiluminated. Top: The divergence between the lights of the objects reilluminated and the rest is $20^{\circ}$. Bottom:The divergence between the lights of the objects reiluminated and the rest is $30^{\circ}$.

Each image was shown to 25 users (ages 17-62, 14 male and 11 female) which were asked the following question: In the following image one or two objects have been inserted and they have a different illumination than the rest of the scene. Could you point it/them out?

$28 \%$ of the users succeeded in spotting one object for the 20 -degree 
image (see Figure 11) whereas, as expected, for 30 degrees of divergence this amount increased up to $36 \%$. Both cases however, are below chance $(40,625 \%$, considering the number of participants that chose one object and the number of participants that chose two). Only one person out of 25 was able to spot both objects, which is slightly above the chance value $(3,125 \%)$.

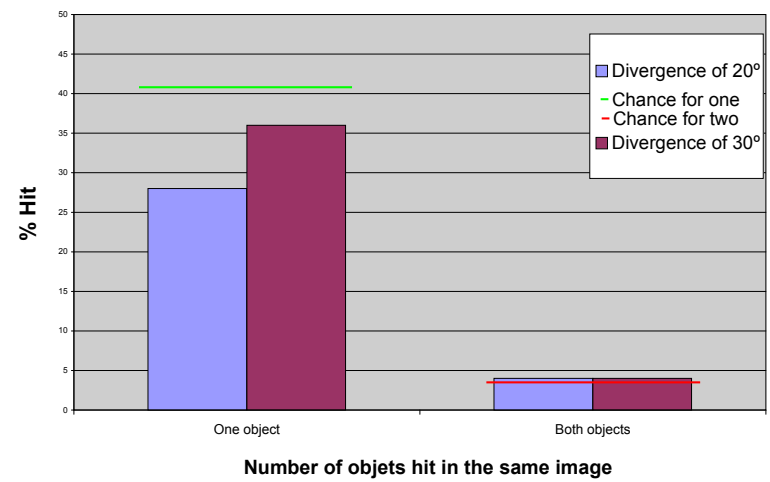

Figure 11: Hit ratio by angle of divergence, grouped by users who spotted correctly one (left) and two objects (right) for both 20 and 30 degrees.

Experiment 3.2 : The test 3.1 was not intended to be exhaustive, but it was designed to give some insight on how conservative a 2030-degree threshold may be in a real-world scenario (in the absence of tell-tale shadows). Our results suggest that it may indeed be overconservative for real images. Our next test aims at generalizing a bit more those findings and it includes objects covering additional materials, textures and shapes; additionally, we extend the range of divergence up to 40 degrees.

In this experiment nine versions of a new scene were generated (See Figure 12). Four photographs of the same scene were taken at 0 , 20, 30 and 40 degrees of divergence from a reference direction. They were combined in the same fashion as in the previous test, but in this case three different objects were masked out and only one object was combined at a time thus obtaining nine versions of the same scene (three objects times three divergence degrees). The black background was used to avoid projection of shadows on a parallel surface and the image composition is done with Poissonbased alpha matting. The result is almost seamless as the local environment of the selected object in both images is very similar.

The objects selected for modification cover a wide range of materials, shapes and positions in the scene: the Santa Klaus doll (diffuse material, high frequency geometry, background position), the metallic robot (Highly specular, rightmost foreground position) and the clown doll (multiple albedo, diffuse, leftmost background position). In total, 60 users (ages 18-59, 38 male and 22 female) took the test. Each user was shown three images with a random inconsistently lit object at 20,30 and 40 degrees of divergence respectively. The same object was never shown more than once per user.

The results of the test (Figure 13) present a similar trend to those from our synthetic experiment, but slightly more conservative: whereas in the synthetic scenes (Experiments One and Two), the detection threshold was somewhere between 20 and 30 degrees, the variety of real world shapes and materials seems to increase that threshold to the 30-40 degree range.
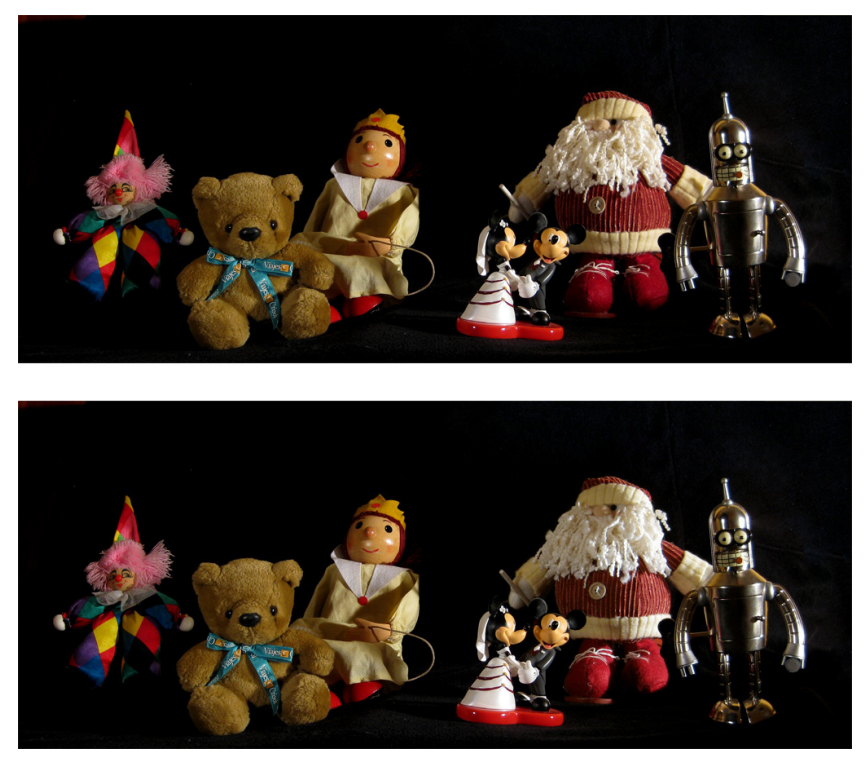

Figure 12: Top:Original image with all the objects consistently lit. Bottom:Example of image used in our experiment. The Santa Klaus doll is lit with a divergence of $\phi=-40$ degrees from the global light direction.

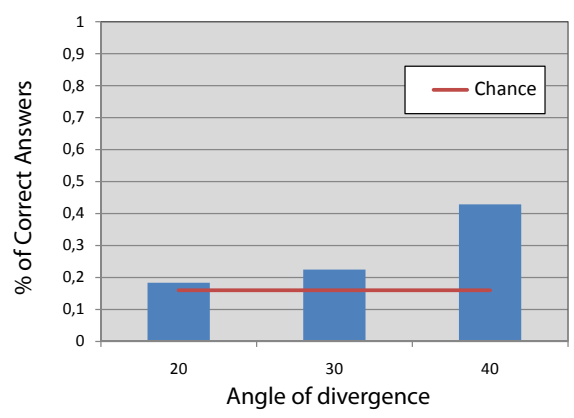

Figure 13: Hit ratio by angle of divergence for 20, 30 and 40 degrees.

\section{Conclusions and Future Work}

We have presented the results of four different tests, whose overall goal was to quantitatively measure the accuracy of human vision detecting lighting inconsistencies in images. We have restricted ourselves to the case of inconsistent light direction. The results of our experiments seem to agree with the theories exposed in previous research on illumination perception [Ostrovsky et al. 2005; Koenderink et al. 2004; Lopez-Moreno et al. 2009], but we have extended those to suggest a perceptual threshold for multiple configurations. Additionally, we have shown how that threshold seems to be even larger for real-world scenes. Although we do not claim our experiments to be exhaustive, we do believe they add significant value to the current state of the art.

We can find several possible interpretations to the fact that lighting inconsistencies were harder to detect in real-world images: it may simply be that the combination of multiple visual cues (texture, shading, highlights...) which was richer than in the CG scenes, might have complicated the detection task. But it is also interesting to dig into the influence produced by the different range of naturalness of the images. 
In similar contexts (3D shape perception) some authors have related naturalness of stimuli to reduced activation in the visual cortex (V1) [Murray et al. 2002; Georgieva et al. 2008], which is related to lowlevel vision. Although the exact relationship between naturalness and the detection process remains unclear, Scott et al.[2002] suggest that under reduced activity in V1 for grouped elements, isolated or novel elements may be more readily detected. There is an apparent contradiction with our results, which might be due to the fact that prior knowledge of 3D shape and material may reduce accuracy. Also, the degree of visual grouping of objects in the synthetic scene (all objects were semantically the same) could have been greater than in the real images (possibly due to increased visual and semantic complexity of individual objects). This might have augmented the tolerance to illumination differences, but in any case it remains a fascinating problem to study.

We believe that the present work may be of value for those areas of computer graphics and vision that depend on analyzing the lighting environment, including algorithms based on light detection and methods for image synthesis (augmented reality...) analysis (digital forgery detection) and processing (special effects). Given that light detection in an image is an ill-posed problem, being able to work within perceptual error thresholds can make the problem tractable.

\section{Acknowledgments}

This research was partially funded by a generous gift from Adobe Systems Inc, the Gobierno de Aragón (projects OTRI 2009/0411 and CTPP05/09) and the Spanish Ministry of Science and Technology (TIN2007-63025).

\section{References}

Adelson, E. H., And Pentland, A. P. 1996. The perception of shading and reflectance. Cambridge University Press, New York, NY, USA, 409-423.

Georgieva, S. S., Todd, J. T., Peeters, R., And Orban, G. A. 2008. The Extraction of 3D Shape from Texture and Shading in the Human Brain. Cerebral Cortex, bhn002.

Hugdahl, K., Thomsenb, T., And Ersland, L. 2006. Sex differences in visuo-spatial processing: an fmri study of mental rotation. Neuropsychologia, 3, 15751583.

Johnson, M. K., AND FARID, H. 2005. Exposing digital forgeries by detecting inconsistencies in lighting. In MM\&Sec '05: Proceedings of the 7th workshop on Multimedia and security, ACM, New York, NY, USA, 1-10.

JOHnson, M. K., AND FARID, H. 2007. Exposing digital forgeries in complex lighting environments. IEEE Transactions on Information Forensics and Security 2, 3, 450-461.

Khang, B., Koenderink, J., And Kappers, A. 2006. Perception of illumination direction in images of 3-D convex objects: Influence of surface materials and light fields. PerceptionLondon 35, 5, 625.

Koenderink, J. J., VAn Doorn, A. J., And Pont, S. C. 2004. Light direction from shad(ow)ed random gaussian surfaces. Perception 33, 12, 1405-1420.

LAGGER, P., AND FUA, P. 2006. Using specularities to recover multiple light sources in the presence of texture. In ICPR '06: Proceedings of the 18th International Conference on Pattern Recognition, IEEE Computer Society, Washington, DC, USA, 587-590.
LANGer, M. S., AND BÜLThofF, H. H. 2001. A prior for global convexity in local shape-from-shading. Perception 30, 403-410.

Lopez-Moreno, J., SAngorrin, F., LATORRe, P., AND GUTIERREZ, D. 2009. Where are the lights? measuring the accuracy of human vision. In CEIG '09: Congreso Español de Informática Gráfica, 145-152.

Mamassian, P., And Goutcher, R. 2001. Prior knowledge on the illumination position. Cognition 81, 1, B1 - B9.

Mingolla, E., AND TodD, J. 1986. Perception of solid shape from shading. Biological Cybernetics 53, 137-151.

Murray, S. O., Kersten, D., Olshausen, B. A., Schrater, P., AND Woods, D. L. 2002. Shape perception reduces activity in human primary visual cortex. Proceedings of the National Academy of Sciences of the United States of America 99, 23, 15164-15169.

O'Shea, J. P., Banks, M. S., And Agrawala, M. 2008. The assumed light direction for perceiving shape from shading. In APGV '08: Proceedings of the 5th symposium on Applied perception in graphics and visualization, ACM, New York, NY, USA, 135-142.

Ostrovsky, Y., Cavanagh, P., And Sinha, P. 2005. Perceiving illumination inconsistencies in scenes. Perception 34, 1301-1314.

Sun, J., AND PERonA, P. 1998. Where is the sun? Nature Neuroscience 1, 3, 183-184.

Todd, J., AND Mingolla, E. 1983. Perception of surface curvature and direction of illumination from patterns of shading. Journal of Experimental Psychology Human Perception and Performance 9, 4, 583-595.

Vangorp, P., Laurijssen, J., And Dutré, P. 2007. The influence of shape on the perception of material reflectance. $A C M$ Trans. Graph. 26, 3, 77.

WANG, Y., AND SAMARAS, D. 2002. Estimation of multiple directional light sources for synthesis of mixed reality images. In Proceedings of the 10th Pacific Conference on Computer Graphics and Applications, 38-47.

Yu, T., Wang, H., Ahuja, N., And Chen, W.-C. 2006. Sparse lumigraph relighting by illumination and reflectance estimation from multi-view images. In Eurographics Symposium on Rendering, Eurographics Association, 41-50.

ZHANG, Y., AND YANG, Y.-H. 2001. Multiple illuminant direction detection with application to image synthesis. IEEE Trans. Pattern Anal. Mach. Intell. 23, 8, 915-920. 